\title{
Más allá del ecocardiograma en el diagnóstico de la insuficiencia cardiaca
}

\author{
Ana Roel Condeㄹ, Miriam Estébanez Muñoz¹, David Martí Sánchez², Carmen González \\ Hernández ${ }^{1}$ \\ 1 Servicio de Medicina Interna, Hospital Central de la Defensa “Gómez Ulla”, Departamento de Medicina y \\ Especialidades Médicas, Universidad de Alcalá de Henares (UAH) \\ 2 Servicio de Cardiología, Hospital Central de la Defensa "Gómez Ulla", Departamento de Medicina y \\ Especialidades Médicas, Universidad de Alcalá de Henares (UAH) \\ * Autor correspondencia: Ana Roel Conde; anaroelconde@gmail.com \\ Recibido: 20/05/2019; Aceptado: 29/05/2019; Publicado: 31/05/2019
}

Resumen: La amiloidosis es una enfermedad sistémica causada por la acumulación extracelular en diferentes tejidos de una proteína mal plegada. Entre los tipos más frecuentes encontramos la transtiretina (TTR) que se subdivide en dos entidades, la TTR mutada que es hereditaria y la TTR wild-type. Cursa con una clínica heterogénea entre la que destaca la insuficiencia cardiaca restrictiva que supone la principal causa de mortalidad. La amiloidosis cardiaca por transtiretina es una entidad infradiagnosticada en la actualidad y requiere una alta sospecha clínica. Presentamos un caso de un paciente de 84 años que ingresa en Medicina Interna por un cuadro de insuficiencia cardiaca descompensada. El ecocardiograma y la RM cardiaca muestran signos de miocardiopatía restrictiva secundaria a amiloidosis que se confirma en el estudio histológico de grasa subcutánea y se establece finalmente el tipo de amiloide mediante la gammagrafía con tecnecio-difosfato. Esta entidad precisa un manejo específico y presenta un pronóstico que difiere de otros tipos de insuficiencia cardiaca.

Palabras Clave: Amiloidosis; Transtiretina; Insuficiencia Cardiaca.

Abstract: Amyloidosis is a systemic disease caused by the extracellular accumulation of a misfolded protein in different tissues. Among the most frequent types of amyloid, we find the transthyretin (ATTR) which is subdivided into two subtypes, the mutated ATTR that is hereditary and the wildtype ATTR. It is characterized by heterogeneous manifestations although the cardiac involvement stands out as the main cause of mortality. Transthyretin cardiac amyloidosis is underdiagnosed and requires a high clinical suspicion. We present a case of an 84-year-old patient admitted to Internal Medicine because of acute heart failure. The echocardiogram and cardiac MRI show signs of restrictive cardiomyopathy secondary to amyloidosis that is confirmed with the histological study of subcutaneous fat and the type of amyloid is finally established by technetium-diphosphate scintigraphy. This entity requires specific management and has a prognosis that differs from other types of heart failure.

Key words: Amyloidosis; Tansthyretin; Heart Failure. 


\section{Introducción}

La amiloidosis es una enfermedad sistémica causada por la acumulación extracelular en diferentes tejidos de una proteína mal plegada, que provoca una alteración de la estructura de distintos órganos y su disfunción [1]. Entre los tipos más frecuentes de proteínas proamiloidóticas encontramos la transtiretina (TTR) o prealbúmina que se sintetiza en el hígado[1]. Existen dos entidades, la TTR mutada que es hereditaria y la TTR wild-type, anteriormente conocida como senil, con fisiopatología desconocida hasta la actualidad y sin mutaciones asociadas [2].

La amiloidosis por TTR cursa con una clínica heterogénea siendo la polineuropatía, el síndrome del túnel del carpo y la insuficiencia cardiaca restrictiva las manifestaciones más frecuentes [2]. Debido a la heterogeneidad de su sintomatología, se establece un amplio diagnóstico diferencial lo que conlleva en muchos casos a un retraso diagnóstico de entre 2 a 3 años, identificándose en estadios avanzados [3]. El manejo terapéutico de estos pacientes es complejo y el tratamiento específico es poco accesible [3].

Presentamos un caso de insuficiencia cardiaca crónica descompensada en el que la alta sospecha clínica y la interpretación adecuada de las pruebas de imagen ponen de manifiesto la importancia de llegar al diagnóstico final de esta entidad que requiere de un manejo específico.

\section{Descripción del caso clínico}

Varón de 84 años con antecedentes de hipertensión arterial, dislipemia, fibrilación auricular anticoagulada con apixaban, insuficiencia cardiaca con disfunción sistólica, insuficiencia renal crónica, gammapatía monoclonal de significado incierto IgG lambda y homocigosis de la mutación H63D del gen HFE. El paciente ingresa en el Servicio de Medicina Interna por sepsis severa de origen biliar y descompensación de insuficiencia cardiaca crónica de predominio derecho.

En la planta de hospitalización se inicia antibioterapia empírica con meropenem iv. con buena respuesta de los parámetros clínicos con remisión de la fiebre y analíticos con disminución de los reactantes de fase aguda. Además, se amplía el diagnóstico etiológico de la cardiopatía. En el ECG se observa una FA con respuesta ventricular rápida y bajo voltaje. El ecocardiograma transtorácico objetiva un aumento severo del espesor de la pared ventricular asociado a FEVI deprimida del $30 \%$ e hipoquinesia global (figura 1a).

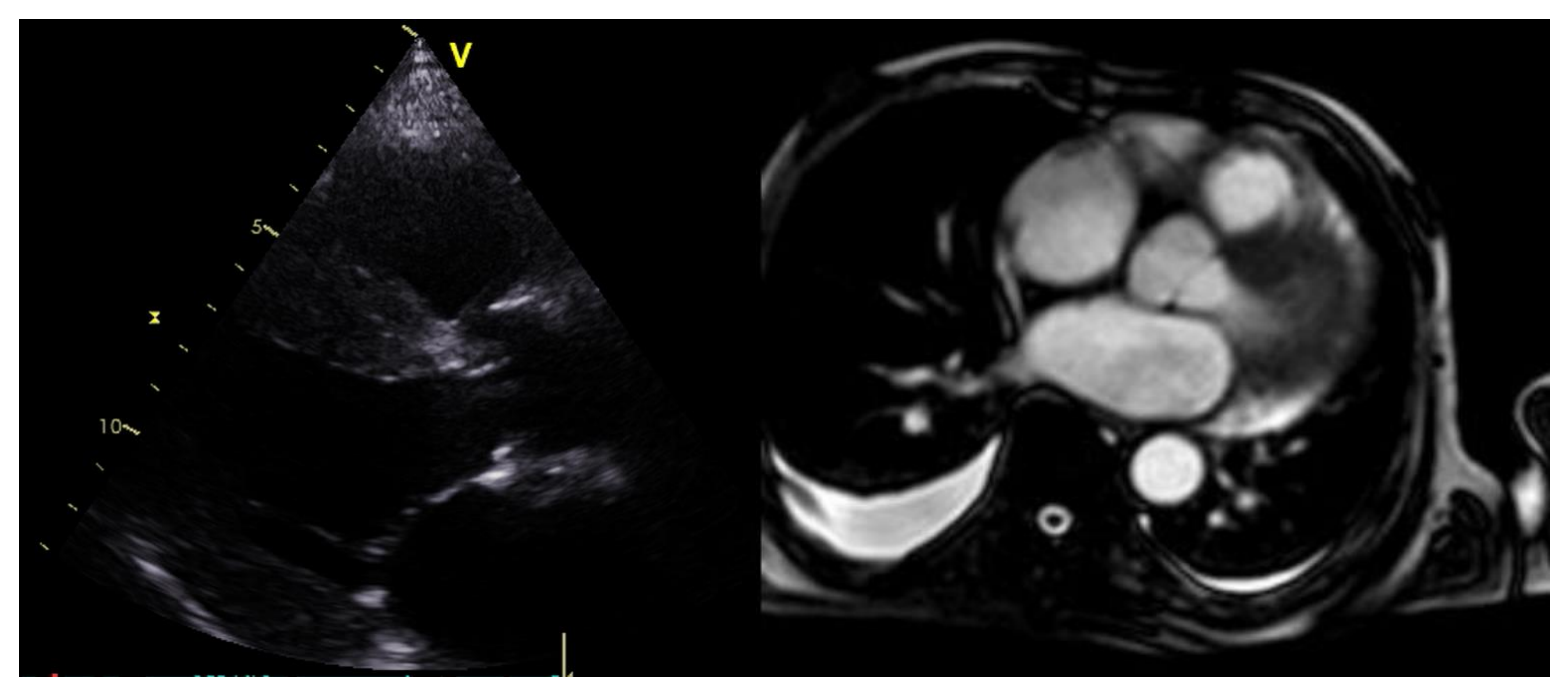

(a)

(b)

Figura 1 (a) Ecocardiograma transtorácico, eje paraesternal largo. Se objetiva hipertrofia concéntrica y patrón granular. (b) RMN cardiaca. Se aprecia realce subendocárdico tardío tras la administración de gadolinio. 
La RM cardiaca confirma el aumento de tamaño de las paredes ventriculares y muestra un realce subendocárdico tardío tras la administración de gadolinio, (Figura 1b) sugestivo de amiloidosis cardiaca. La biopsia de grasa subcutánea de pared abdominal identificó el depósito de amiloide con la tinción de Rojo Congo.

Tras el diagnóstico histológico de amiloidosis, se solicita una batería de pruebas para despistaje de afectación de otros órganos y para determinar el tipo de amiloide. El electromiograma objetiva la presencia de síndrome de túnel carpiano y una polineuropatía sensitivo-motora de carácter mixto y axonal desmielinizante de grado moderado. El estudio inmunológico constata una banda monoclonal de Ig G con 1.2 g/dl, con el índice Kappa/lambda en suero de 0,87 (rango normal: 0,26-1,65). Se completa estudio con una gammagrafía con tecnecio difosfato (99mTc-DPD) que muestra una captación intensa (grado 3 de Perugini) [3] del trazador exclusivamente a nivel cardiaco (Figura 2) lo que confirma el diagnóstico de amiloidosis cardiaca por TTR. El estudio genético discrimina entre TTR mutada o senil, pendiente de resultado en nuestro caso.

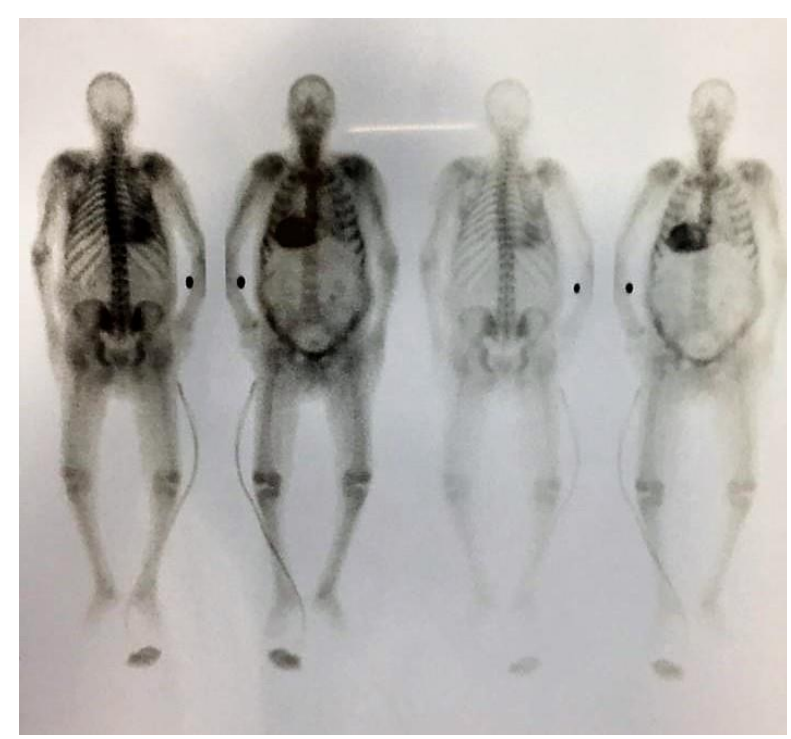

Figura 2 Gammagrafía 99mTc-DPD.Muestra una captación cardiaca grado 3.

\section{Discusión}

Presentamos un caso clínico de insuficiencia cardiaca descompensada de predominio derecho como forma de presentación de una amiloidosis sistémica por TTR.

La amiloidosis por TTR es una entidad infradiagnosticada en la actualidad. Hasta un 5\% de los casos con diagnóstico de miocardiopatía hipertrófica en mayores de 55 años son realmente secundarios a amiloidosis cardiaca por TTR [4]. La afectación cardiaca es la principal causa de mortalidad en los dos tipos de amiloidosis por TTR, la senil y la hereditaria. Se estima que el síndrome del túnel carpiano puede llegar a predecir la cardiopatía hasta 6 años antes [5]. El registro THAOS europeo 2019 describe afectación cardiaca en un 36\% de los pacientes con amiloidosis por TTR, independientemente del tipo. El 58\% de los pacientes con amiloidosis cardiaca por TTR presentan afectación neurológica concomitante tanto en la forma hereditaria como en la senil, siendo obligado su despistaje [6].

La sospecha clínica de insuficiencia cardiaca secundaria a amiloidosis por TTR se establece en pacientes varones, mayores de 55 años, sin hipertensión arterial, con hipertrofia simétrica de VI y VD y patrón granular en el ecocardiograma [6]. Clásicamente, la confirmación diagnóstica se establece mediante la visualización histológica del material amiloide. El gold estándar en el diagnóstico de amiloidosis cardiaca por TTR es la biopsia endomiocárdica [2]. Es un método invasivo que precisa de experiencia y no evalúa la extensión sistémica del material amiloide [7]. Está indicada cuando no se consigue demostración extracardiaca o en amiloidosis cardiaca localizada [2]. Esta técnica se rechaza con frecuencia debido a la dificultad y riesgo de complicaciones en pacientes con insuficiencia cardiaca avanzada [7]. Actualmente, se emplea un algoritmo diagnóstico no invasivo con la gammagrafía con tecnecio difosfato (99mTc-DPD) [3] que utiliza un trazador óseo altamente sensible para ambos tipos de amiloidosis por TTR. Se evalúa la captación mediante la escala de Perugini alcanzando una sensibilidad cercana al 100\% si score 3 [4] como ocurre en este paciente. En este caso, el paciente presentaba una gammapatía monoclonal de significado incierto con un índice kappa/lambda normal, lo que hacía poco probable la afectación cardiaca por amiloidosis por cadenas ligeras (AL). En ancianos, un incremento moderado de cadenas ligeras circulantes no debe llevar directamente al diagnóstico de amiloidosis AL [3]. 
Tras el diagnóstico de amiloidosis por TTR está indicado en todos los casos el estudio genético, que permite discriminar entre TTR mutada y TTR wild-type para consejo familiar [6].

El tratamiento fundamentalmente es de soporte y difiere de las recomendaciones generales del tratamiento de la insuficiencia cardiaca. Los IECAS son mal tolerados al causar hipotensión. La digoxina y los antagonistas del calcio están contraindicados porque se acumulan en el tejido amiloide [2]. El trasplante hepático es el único tratamiento curativo en TTR mutada [3]. Estudios en fase 3 describen un nuevo fármaco, el tafamidis, que actúa evitando el mal plegamiento de la proteína y que ha demostrado reducir la mortalidad global en un 13\%, disminuir el número de hospitalizaciones y frenar el empeoramiento de la capacidad funcional y calidad de vida en estos pacientes [8].

El pronóstico se establece mediante una clasificación específica desarrollada por la Clínica Mayo que tiene en cuenta los niveles de proBNP $(>3.000 \mathrm{pg} / \mathrm{ml})$ y de troponina $\mathrm{T}(>0,05 \mathrm{ng} / \mathrm{ml})$ [3]. En la actualidad la mayoría de los pacientes se diagnostican en estadio III con una supervivencia de 20 meses que contrarresta con los 66 meses del estadio I como es el caso de este paciente [3].

\section{Conclusiones}

La amiloidosis cardiaca por TTR es una patología prevalente que requiere una alta sospecha clínica al cursar con síntomas inespecíficos. Las nuevas técnicas diagnósticas no invasivas permiten un diagnóstico precoz.

El tratamiento de soporte de esta afección cardiaca difiere del tratamiento de la insuficiencia cardiaca secundaria a cardiopatía hipertensiva, entidad con la que se suele confundir.

El análisis genético debe realizarse en aquellos pacientes en los que se haya confirmado la amiloidosis por TTR, independientemente de la edad, para poder realizar el consejo genético familiar.

Se han desarrollado nuevos fármacos con intención curativa, aún con limitadas indicaciones, que pueden modificar el pronóstico de estos pacientes.

Contribución de los autores: Los autores han contribuido al diagnóstico y tratamiento de la paciente así como a la revisión del manuscrito.

Conflictos de Intereses: Los autores declaran que no tienen conflictos de interés.

\section{Abreviaturas}

Las siguientes abreviaturas son usadas en este manuscrito:

TTR: transtiretina

AL: amiloidosis por cadenas ligeras

VI: ventrículo izquierdo

VD: ventrículo derecho

\section{Referencias Bibliográficas}

1. Nienhuis H, Bijzet J, Hazenberg B. The Prevalence and Management of Systemic Amyloidosis in Western Countries. Kidney Diseases. 2016;2:10-19

2. García-Pavía, P., Tomé-Esteban, M. and Rapezzi, C. Amiloidosis. También una enfermedad del corazón. Revista Española de Cardiología 2011;64: 797-808.

3. González-López E, López-Sainz Á, Garcia-Pavia P. Diagnóstico y tratamiento de la amiloidosis cardiaca por transtiretina. Progreso y esperanza.." Revista Española de Cardiología. 2017; 70: 991-1004.

4. Culotta V, Moon J. Amiloidosis cardiaca por transtiretina: antes una enfermedad monogénica minoritaria, ¿ahora una vía común en la insuficiencia cardiaca? Revista Española de Cardiología. 2016; 69:888-889

5. Galant N, Westermark P, Higaki J, Chakrabartty A. Transthyretin amyloidosis: an under-recognized neuropathy and cardiomyopathy. Clinical Science. 2017;131:395-409.

6. Damy T, Kristen A, Suhr O, Maurer M, Planté-Bordeneuve V, Yu C et al. Transthyretin cardiac amyloidosis in continental Western Europe: an insight through the Transthyretin Amyloidosis Outcomes Survey (THAOS). European Heart Journal. 2019; 0, 1-10 
7. Bokhari S, Castaño A, Pozniakoff T, Deslisle S, Latif F, Maurer MS. "Bokhari S, Castaño A, Pozniakoff. $(99 \mathrm{~m}) \mathrm{Tc}-$ pyrophosphate scintigraphy for differentiating light-chain cardiac amyloidosis from the transthyretin-related familial and senile cardiac amyloidoses." Circ Cardiovasc Imaging 2013; 6(2):195-201

8. Maurer M, Schwartz J, Gundapaneni B, Elliott P, Merlini G, Waddington-Cruz M et al. Tafamidis Treatment for Patients with Transthyretin Amyloid Cardiomyopathy. New England Journal of Medicine. 2018; 379:1007-1016.

(C) 2019 por los autores; Esta obra está sujeta a la licencia de Reconocimiento 4.0 Internacional de Creative Commons. Para ver una copia de esta licencia, visite http://creativecommons.org/licenses/by-nc-nd/4.0/. 